\title{
Efectividad metodológica para el levantamiento de requerimientos de aplicaciones web
}

Fecha de recepción: 11 de octubre de 2016

Fecha de aprobación: 20 de diciembre de 2016

Pp. 71-98
César Augusto López Caballero ${ }^{I}$ Universidad Autónoma de Bucaramanga cesarlopezc@hotmail.com

Silvio Rafael Cuello de Ávila ${ }^{2}$ Universidad Autónoma de Bucaramanga scuellod@unab.edu.co

DOI: https://doi.org/10.21158/23823399.v4.n2.2016.1628

Cómo citar este artículo/ To reference this article/ Comment citer cet article/ Para citar este artigo: López, C. A.; Cuello de Ávila, S. (2016). Efectividad metodológica para el levantamiento de requerimientos de aplicaciones web. Revista Ontare, 4(2), 71-98 .DOI: https://doi. org/10.21158/23823399.v4.n2.2016.1628

\section{Resumen}

El presente proyecto busca evaluar la efectividad del proceso de investigación de requerimientos, mediante una metodología compuesta por cinco fases (elicitación, modelado, codificación, comprobación -análisis, verificación y validación- e impresión de uso), y dos herramientas (un formato de preguntas agrupadas por aspectos generales y un prototipo o modelo de pantalla). En lo que respecta a las fases, las dos primeras corresponden a la propuesta central y las tres siguientes a la estructura de cuantificación. En cuanto a las herramientas, el formato está estructurado en cinco aspectos generales: fundamentación, tiempos, organización institucional, negocio y aspectos sistémicos y el prototipo de pantalla nace como resultado del entendimiento de los requerimientos preliminares establecidos.

\section{Palabras-clave}

Aplicaciones, efectividad, satisfacción, metodologías, requerimientos.

\footnotetext{
${ }^{1}$ Licenciado en Informatica (Universidad Minuto de Dios). Magister en software libre (Universidad Autónoma de Bucaramanga). ORCID: http://orcid.org/0000-0002-4387-8660

2 Estudiante del programa de Ingeniería mecânica, de la universidad UNIVATES de Brasil.
} 


\title{
Methodological effectiveness for the elicitation of web application requirements
}

\begin{abstract}
The present project seeks to evaluate the effectiveness of the requirements research process, using a methodology composed of five phases (elicitation, modeling, coding, testing - analysis, verification and validation - and impression of the use), and two tools (a format including questions that are grouped by general aspects and a prototype or model of screen). As regards to the phases, the first two correspond to the central proposal and the other three to the quantification structure. As for the tools, the format is structured in five general aspects: fundamentals, times, institutional organization, business and systemic aspects, and the prototype screen is born as a result of the understanding of the established preliminary requirements.
\end{abstract}

\section{Keywords}

Applications, effectiveness, satisfaction, methodologies, requirements.

\section{Effectivité méthodologique pour la levée de requêtes d'applications web}

\section{Résumé}

Ce projet cherche à évaluer l'effectivité du processus de recherche de requêtes au moyen d'une méthodologie composée de cinq phases distinctes (élicitation, modèle, encodage, vérification - analyse, vérification et validation - et impression d'usage) et deux outils (une fiche de questions regroupant les aspects généraux et un prototype ou modèle d'écran). En ce qui concerne les phases, les deux premières correspondent à la proposition centrale et les trois suivantes à la structure de quantification. Pour ce qui est des outils, le format est structure en cinq aspects généraux: fondements, temps, organisation institutionnelle, affaire et aspects systémiques. Le prototype de d'écran naît donc du résultat de la compréhension des requêtes préliminaires établies.

\section{Mots clefs}

Applications, effectivité, satisfaction, méthodologies, requêtes. 


\section{Efetividade metodológica para o levantamento de requerimentos de aplicações web}

\section{Resumo}

O presente projeto procura avaliar a efetividade do processo de investigação de requerimentos, mediante uma metodologia composta por cinco fases (elicitación, modelagem, codificação, comprovação-análise, verificação e validação - e impressão de uso), e duas ferramentas (um formato de perguntas agrupadas por aspectos gerais e um protótipo ou modelo de tela). No relacionado às fases, as duas primeiras correspondem à proposta central e as três seguintes à estrutura de quantificação. Quanto às ferramentas, o formato está estruturado em cinco aspectos gerais: fundamentação, tempos, organização institucional, negócio e aspectos sistêmicos e o protótipo de tela nasce como resultado do entendimento dos requerimentos preliminares estabelecidos.

\section{Palavras-chave}

Aplicações, efetividade, satisfação, metodologias, requerimentos. 


\section{Introducción}

e la organización en el proceso de levantamiento de requerimientos depende el éxito y los buenos resultados en la implementación de las aplicaciones (Terstine, 2015), el generar una estructura que organice las respuestas y necesidades sistémicas de los interesados, garantizan la proyección y el modelado de los requerimientos (Pérez, Salamando, y Valencia, 2012), haciendo posible que la efectividad aumente en las metodologías propuestas $\mathrm{y}$, por consiguiente, aumente la satisfacción en los clientes o usuarios. El presente ejercicio de investigación se desarrolló en un contexto académico de cuatro instituciones distritales administradas por la Caja de Compensación Familiar CAFAM en Bogotá, D.C, las cuales están enmarcadas en la educación formal desde los niveles de primero hasta grado once de educación media, en donde los profesores realizan procesos de planeación pedagógica en todas las asignaturas o áreas del conocimiento.

El presente proyecto parte del objetivo general que es evaluar la efectividad del proceso de investigación de requerimientos utilizando dos herramientas: un conjunto de preguntas sincronizadas o agrupadas por aspectos generales y un prototipo o modelo de pantalla, el cual es el resultado del entendimiento de los requerimientos preliminares de los interesados. Ahora bien, los aspectos generales son herramientas de análisis semántico propuestas para este trabajo, que está compuesto por cinco aspectos que engloban un conjunto de preguntas previamente formuladas, con las cuales se puede extraer información relacionada con la fundamentación, tiempos, organización institucional, aspectos de negocio y, finalmente, aspectos sistémicos de la aplicación.

Con referencia al objetivo general y a la organización del artículo, se plantea una estructura de cuatro objetivos específicos que inician con la formulación y aplicación de una secuencia de preguntas para obtener la caracterización relacionada con tiempos, fundamentación 
pedagógica, organización institucional y periodicidades del proceso de la planeación de la acción pedagógica, posteriormente se continúa con el objetivo específico relacionado con el diseño de los prototipos de pantallas, la validación de estos y la creación del modelo entidad relación, continuando con el objetivo específico vinculado con la codificación de los requerimientos con base en la información y los modelos elicitados. Finalmente, se procede a desarrollar el objetivo específico relacionado con la evaluación de la efectividad en el levantamiento de los requerimientos y satisfacción del uso de la aplicación web por parte de los usuarios.

Como respuesta a la necesidad del anterior contexto y objetivos del proceso de investigación, se enmarca el objetivo con la medición de la efectividad, utilizando las herramientas mencionadas e iniciando con el conocimiento del dominio o modelo de planeación pedagógica a través de una entrevista estructurada (Schach, 2006); posteriormente, acude a técnicas de corta distancia y multitudes en ingenieria del software, en donde propone una estructura conceptual materializada en un instrumento de veinticinco (25) preguntas agrupadas por cinco (5) aspectos generales, las cuales permiten captar, organizar y entender la informacion recogida en el conocimiento del dominio (Terstine, 2015).

Luego se analiza un método para la extracción de requerimientos usando la minería de textos en correos electrónicos (Alvarado y Cuervo, 2013), pero adaptado al análisis semántico de las respuestas que entregan los usuarios del contexto de estudio y relacionándolos con el mundo de las entidades reales y abstractas de las bases de datos a través de una serie de aspectos generales, migrando estas respuestas a requerimientos y estos, a su vez, a las entidades del mundo real y modelos entidad relación (Pérez et al., 2012).

El ejercicio de investigación inició con el modelado del prototipo de pantalla con los conocimientos previos sobre el dominio por parte del primer autor (Mansilla et al., 2012), pero la esencia del modelo propone iniciar con el diseño del instrumento (preguntas con rangos orientadores 
relacionadas con el universo de las entidades abstractas y reales de las bases de datos) y, posteriormente, la aplicación de este, el análisis a profundidad de las respuestas, la obtención de los requerimientos preliminares, la configuración del prototipo propuesto y, finalmente, la validación del prototipo de pantalla hasta obtener el mejor entendimiento de los requerimientos plasmados en el modelo, el cual servirá de norte en las fases siguientes (Figura 1).

El objetivo del uso del modelo o prototipo de pantalla consiste en canalizar el entendimiento y lenguaje técnico en los procesos de elicitación (Manies \& Nikual, 2011), y el diseño y aplicación del formato de preguntas consiste en generar una estructura para extraer características necesarias en el diseño y construcción de una aplicación, garantizando así la correcta proyección de los requerimientos (Anaima Dasilva, 2015).

Figura 1. Diagrama metodología para el levantamiento de requerimientos.

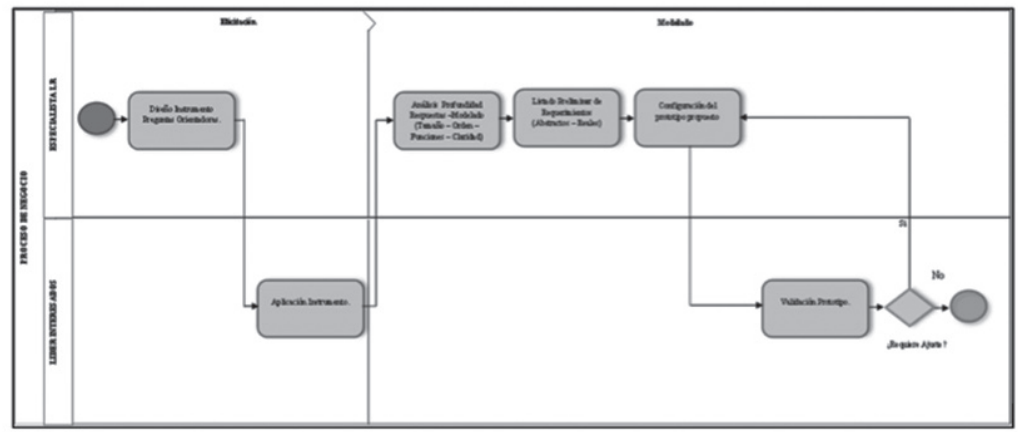

Fuente. Elaboración propia. 


\section{Desarrollo del artículo}

T a propuesta central de la metodología está compuesta por dos fases: elicitación y modelado, las tres fases posteriores corresponden con la estructura de cuantificación. Estas dos fases iniciales combinan aspectos relacionados con procesos de abstracción de las respuestas elicitadas a requerimientos y estos a entidades y a modelos entidad relación. Posteriormente, se ejecutan las fases de codificación, comprobación e impresión de uso, para obtener los indicadores de efectividad y satisfacción (Figura 2).

En la fase de comprobación se obtiene un indicador de efectividad del entendimiento de los requerimientos a partir de la comparación entre los requerimientos preliminares y las observaciones que surgen en la revisión de los líderes con la aplicación web desarrollada. La justificación de este indicador nace en el éxito de la metodología, es decir, si los requerimientos fueron plasmados en la aplicación y estos no tuvieron ninguna observación en el segundo momento, la efectividad de la metodología es óptima porque no genera reprocesos en las fases de elicitación, modelado y codificación. Finalmente, se muestra cómo se realizó el proceso de la valoración de la impresión de uso de la herramienta, de donde se obtiene un indicador de satisfacción de la muestra seleccionada.

En los siguientes literales del presente numeral se mostrará el proceso y resultado de cada fase de la metodología en términos cuantitativos y operativos, en el numeral III se relacionarán las conclusiones y recomendaciones para futuros trabajos de investigación. 
Figura 2. Diagrama metodología complementaria para el levantamiento de requerimientos. La sigla MER significa Modelo Entidad Relación.

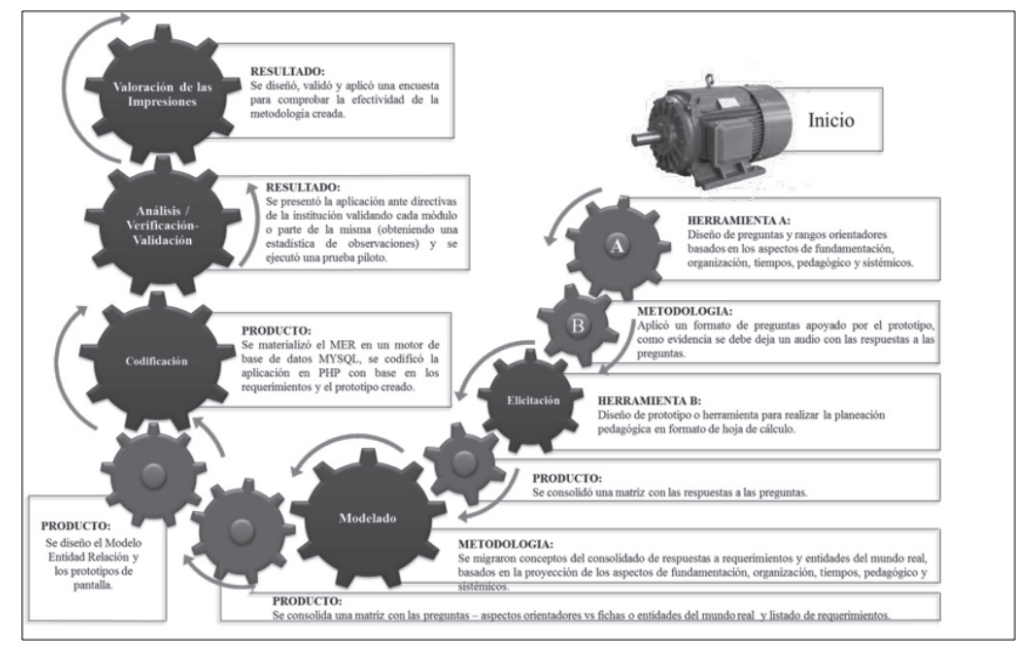

Fuente. Elaboración propia.

\subsection{Fase de elicitación}

En esta fase se debe obtener la percepción de los líderes acerca de la definición conceptual o la necesidad sistemática de la aplicación por desarrollar, para lo cual esta metodología plantea la creación de un formato de preguntas, que se orientan hacia la consecución sistemática de las características reales o abstractas de las bases de datos. En las siguientes líneas se describirá las características del proceso de construcción del instrumento y su proceso de aplicación, el cual entrega una matriz con las respuestas para la fase de modelado.

El proceso de construcción de este formato de preguntas inicia con la definición de los aspectos generales, los cuales se constituyen en unos pilares investigativos que hacen que las preguntas tengan un eje en común, que cobren relevancia y que sean objetos capaces de entregar información relacionada con la importancia, orden y claridad dentro de un conjunto de postulados, que hacen que sirvan de estructura y 
parámetro general en la formulación de estos. Es importante destacar que el modelo propuesto está compuesto por cinco aspectos, los cuales no tienen un orden predefinido; sin embargo, el especialista debe buscar ese orden lógico y el número de preguntas que lo componen.

El presente modelo inicia con el aspecto de fundamentación, es decir, la información relacionada con los objetivos y definición conceptual de las actividades; el aspecto de tiempo indaga por todas las periodicidades, mirando las proyecciones en tiempo y aspectos que nos dan el imaginario de rutinas y cantidad de veces que se repite un evento o actividad; en el aspecto organización institucional, se indaga por la estructura de orden jerárquico con sus respectivas características. Aquí se busca obtener información de carácter organizacional o administrativo, estableciendo características de parentesco, nivel y orden.

En lo relacionado con la actividad en donde actuará la aplicación, se indaga por la organización conceptual, para obtener una radiografía de los componentes y subcomponentes, trazando un hilo conductor con el aspecto de tiempo y estructuras jerárquicas, es decir, une los aspectos reales con los abstractos. Finalmente, en el aspecto sistémico se indaga por los beneficios que debe brindar la herramienta, en este aspecto es importante hacer hincapié en las bondades y cómo funcionará la nueva aplicación, qué restricciones, qué limitaciones tendrá, este es el punto de inicio y fin del desarrollo de la aplicación, son las características que se deben cumplir a cabalidad.

Ahora, teniendo claridad de la estructura de los aspectos orientadores, posteriormente se procede con la formulación de las preguntas, cada una pretende recabar información con su aspecto relacionado, buscando siempre obtener el máximo de conocimiento del negocio, generando así la seguridad del entendimiento de las necesidades sistémicas de la organización y el posterior desarrollo de la aplicación tabla 1. 
Tabla 1. Formato de preguntas orientadoras utilizado en el proyecto.

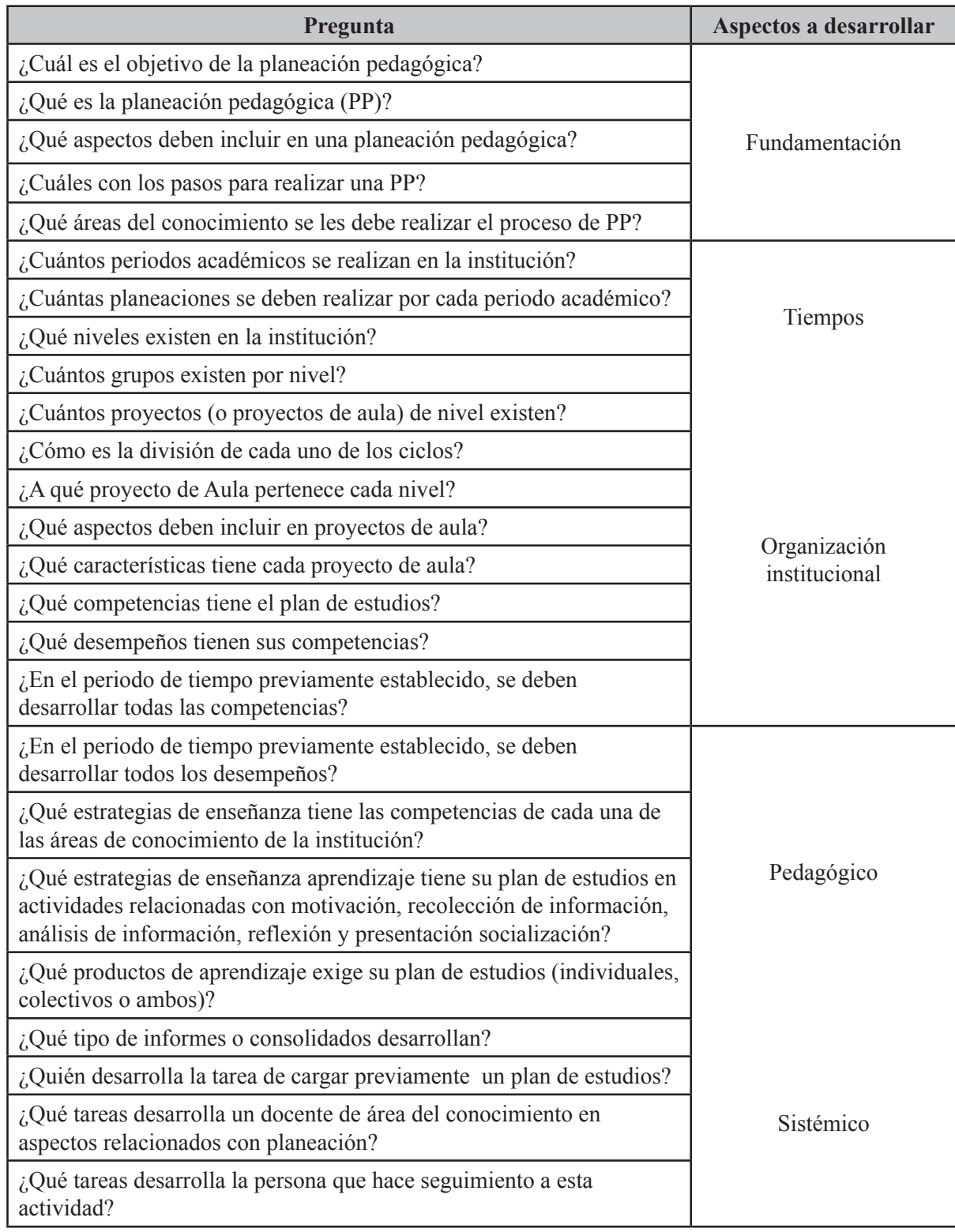

\section{Fuente. Elaboración propia.}


En este estadio de la metodología se aplica el formato de preguntas por medio de una entrevista al líder de los interesados y diferentes miembros, la cual puede ser grabada previa autorización del entrevistado y posteriormente transcrita en una matriz compuesta por las preguntas, las respuestas y el aspecto orientador, que es el insumo principal y único de la siguiente fase.

\subsection{Fase de modelado}

En esta fase el especialista en levantamiento de requerimientos analiza a profundidad la matriz anterior y obtiene las preguntas y respuestas de carácter abstracto y real, visualizando el orden, formas de organización, relación entre cada respuesta y su tamaño e importancia ante las demás. En este sentido, las preguntas y sus respuestas de carácter abstracto se pueden relacionar con todas aquellas que representan características propias del negocio, como sus reglas, tiempos, estructuras de componentes y subcomponentes, maneras de abordar la solución a problemas. Las preguntas y respuestas de carácter real muestran los actores que representan condiciones tangibles (humanos) y cómo estos entran a enlazarse con esos constructos abstractos de la primera parte y, finalmente, cómo se relaciona lo abstracto con lo real.

En síntesis, el producto que entrega este momento es la clasificación de esas preguntas y respuestas que representan algo concreto, bien sea real o abstracto, pero lo entrega caracterizado o clasificado especificando un orden, un tamaño, una jerarquía y unas funciones determinadas, de manera que se pueda describir un requerimiento incluyendo las anteriores características.

Teniendo claro la importancia de orden y claridad de las respuestas abstractas y reales, se procede a migrar la información de las respuestas a la lista de requerimientos, mirando esos comunes denominadores y cómo 
se podrían visualizar en un requerimiento que coadyuve a mejorar el entendimiento de las necesidades sistémicas de los usuarios y el posterior desarrollo de la aplicación.

La acción real para obtener los requerimientos es la organización de las matrices. Es la forma de tener la visual de la información compuesta por la pregunta y la respuesta en su orden correspondiente. Posteriormente, se buscan los actores que están inmersos en ese contexto, teniendo en cuenta sus relaciones de pertenencia que se traducen en integridades referenciales. Seguido de este momento se puede ver la constitución de esos campos que entrarán a formar parte de ese primer prototipo, el cual debe ser validado y aceptado por los líderes de los interesados.

Posteriormente, para generar la lista de requerimientos se recurre a la escritura de textos que incluyan los actores (ya sean abstractos o reales) y sus relaciones, funciones y comportamientos en la aplicación por desarrollar, mostrando los niveles de integridad, relaciones con los otros actores abstractos o reales. En la columna de requerimientos se puede ratificar la importancia, orden y claridad conceptual de sus actores abstractos entre niveles y grupos (entidad real), es decir, un nivel puede contener un número indeterminado de grupos, en el segundo requerimiento se evidencia una relación entre proyecto de aula y grupos (Tabla 2). 
Tabla 2. Desarrollo del consolidado de respuestas al listado de requerimientos a entidades y diagrama organización institucional.

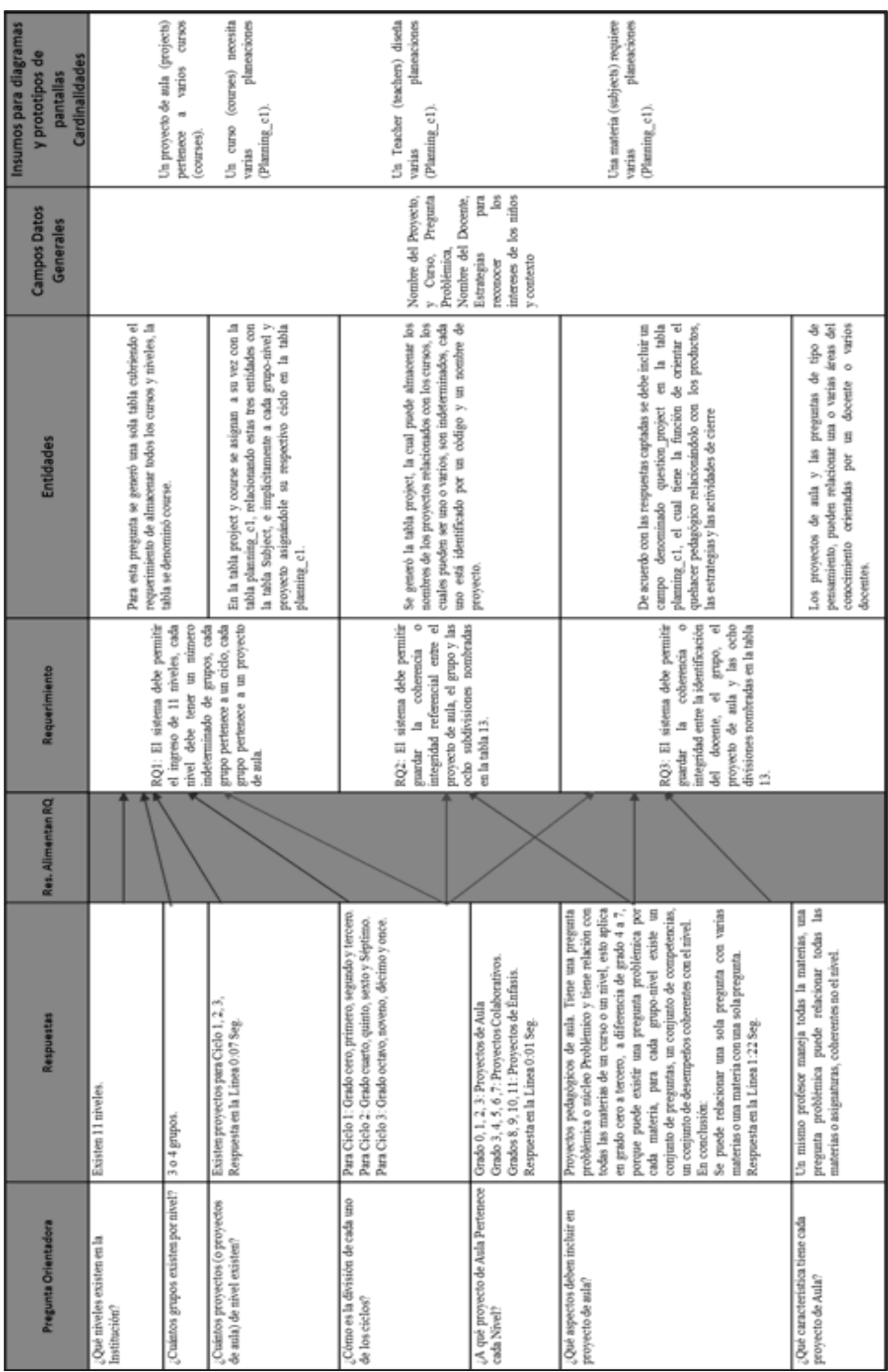

Fuente. Elaboración propia. 
El proceso para conformar los requerimientos se basa en la unión de las respuestas, en el ejemplo se orienta por las líneas de colores: para el RQ N. ${ }^{\circ} 1$ lo conforman las respuestas N. ${ }^{\circ} 1$ a la N. ${ }^{\circ} 4$, al analizar estas preguntas y sus respuestas se pueden abstraer que existen once niveles, que cada nivel está compuesto por un número indeterminado de grupos y que cada ciclo está dividido en grados y que a cada ciclo le corresponde un proyecto de aula. En el RQ N. ${ }^{\circ} 2$ se puede abstraer que solicita la integridad referencial entre ciclos, grupos, proyectos de aula y las especificaciones restantes ${ }^{3}$. Finalmente, en el RQ N. ${ }^{\circ} 3$ se puede observar que solicita la integridad entre el docente, ciclos, proyecto de aula y asignaturas (Tabla 2).

El proceso para conformar las entidades se puede observar en la relación entre la columna de respuestas y entidades, existen siete preguntas, pero de la primera a la tercera conforman un comentario de entidad, la cuarta y la quinta forman otro comentario. El valor agregado de este ejercicio es identificar cómo se analiza la entidad que inicia con la cadena de pertenencia ${ }^{4}$ y cómo se puede organizar la información para tener la mejor comprensión y claridad.

Continuando con el párrafo anterior, lo primero que se debe preguntar el especialista es qué actores están implícitos en los requerimientos; en este ejemplo los requerimientos abstractos se pueden visualizar en la organización administrativa materializada en los (1) niveles, (3) ciclos, (4) conformación de los ciclos, (5) proyectos de aula, y los actores reales están representados por grupos (2) y docente.

3 Listado de especificaciones: Datos generales, pregunta por tipo de pensamiento, competencias y desempeños, Estrategias de enseñanza aprendizaje, Ruta metodológica, Productos de aprendizaje, Cierre de proyecto, Enfoque diferencial, Observaciones Coordinación.

4 La cadena de pertenencia se define como la entidad que agrupa con mayor definición a otras entidades. 
Posteriormente, el especialista en levantamiento de requerimientos debe buscar la mejor forma de personalizar los actores abstractos y reales, debe buscar el mejor campo para identificarlos y utilizar el principio de filtros (los utilizados en hojas de cálculo), en donde se va reduciendo columna a columna hasta obtener la información que se busca visualizar. Los campos por incluir en el modelo se pueden visualizar en la columna datos generales (tabla 2).

La actividad restante consiste en crear la representación gráfica con los campos mencionados, teniendo en cuenta el orden y la importancia de lo abstracto y lo real; por ejemplo, en lo real aparecen los campos nombre del docente y asignatura. Dentro de lo abstracto figuran nombre del proyecto, pregunta problema, estrategias para reconocer intereses de los niños, asignatura, periodo y fecha (Figura 3 ).

Figura 3. Prototipo Datos generales PAP.

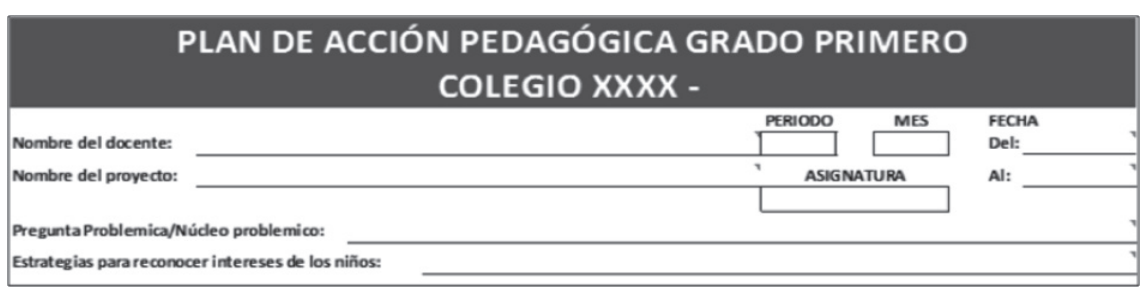

Fuente. Elaboración propia.

Para cerrar y entregar los resultados a otras fases del proyecto, se gestiona un encuentro de validación y aceptación de los líderes interesados, para revisar a la luz de los requerimientos y sus antecedentes la calidad del prototipo de pantalla, posteriormente aprobando o solicitando ajustes hasta lograr su calidad.

Finalmente, se puede concluir que el uso de estas técnicas abstractas y reales maquetadas a través de unos aspectos orientadores, operados por unas preguntas, garantiza la proyección adecuada de la aplicación $y$, por ende, reduce los riesgos de reprocesos en los flujos de trabajo 
del programador. Esto aumenta la efectividad del entendimiento de los requerimientos elicitados y lo codificado en la aplicación.

En este momento se había validado el norte de las acciones, para continuar con la migración de requerimientos a entidades y el diseño de la base de datos de la aplicación. Para esto se recurre a las técnicas de normalización y a los siguientes postulados: la entidad que inicia con la cadena de pertenencia de acuerdo con las respuestas entregadas por los interesados y la información de mayor a menor o viceversa, es decir, cómo se puede organizar la información de manera que se pueda obtener más claridad.

Validando los pasos anteriores, en la columna entidades se puede encontrar que el usuario en sus respuestas nombra conceptos de ciclos, niveles, grupos, proyectos de aula y planeación. Ahora bien, en las dos primeras respuestas relaciona los niveles con los grupos, por lo tanto, se propone una primera entidad denominada courses, la cual tiene la función de almacenar los niveles y grupos. Se hace claridad que existen o se proponen tres tipos de proyectos para los ciclos 1,2 y 3, por lo tanto, se propone la entidad projects, la cual tiene la función de almacenar los nombres de los proyectos de todos los ciclos, posteriormente en la cuarta respuesta se agrupan los grados por cada ciclo, y en la sexta respuesta se relacionan los conceptos relacionados con courses, projects y planeación, por consiguiente, se genera la entidad planinng_c1, en donde termina la primera cadena de pertenencia.

Finalizando la descripción de la migración de las respuestas a la propuesta de entidades, se puede apreciar que la entidad projects reúne o le pertenecen los ciclos, niveles y grupos plasmados en la entidad courses, y este a su vez se relaciona con la entidad planning_c1; hay que puntualizar que el aspecto de ciclo queda mediado en la entidad de planning_cl por tener la función de almacenar solo información para ciclo 1 (Figura 4). 
Figura 4. Modelo Entidad Relación y Ruta de creación de entidades Paponline.

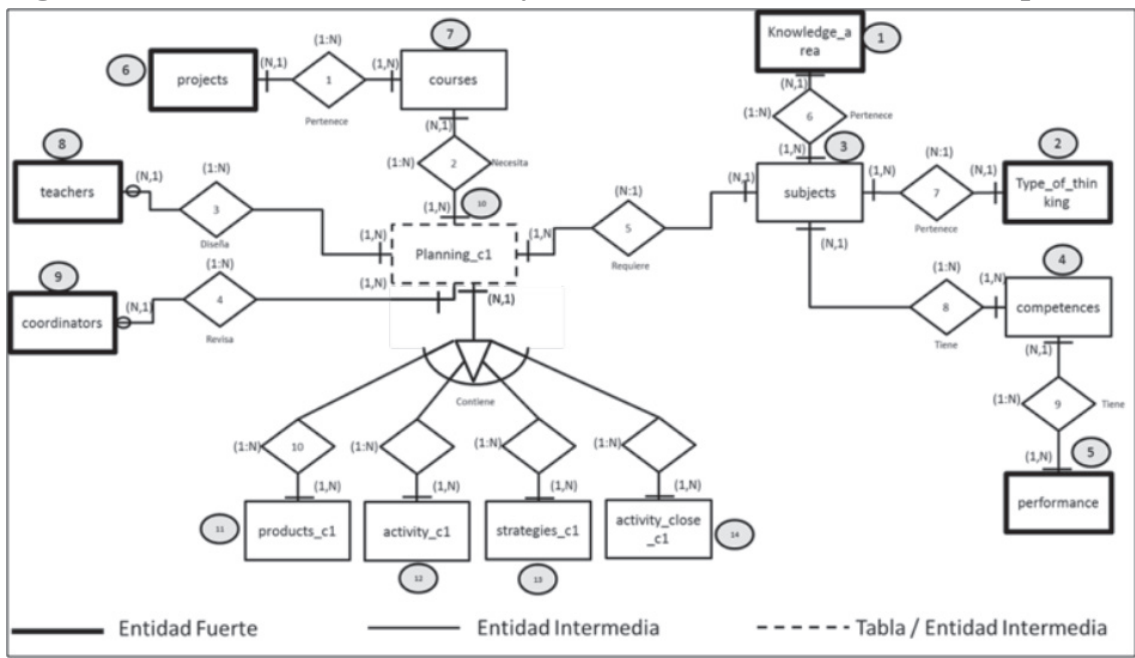

Fuente. Elaboración propia.

\subsection{Fase de codificación de bases de datos y formularios}

Este proceso se inició con la revisión de las cardinalidades en los diagramas de la fase anterior, después se seleccionó el motor de base de datos MYSQL, el cual pertenece a la familia de software denominada XAMPP Control Panel V3.2.2, con un servidor de base de aplicaciones WEB Apache/2.4.17, PHP 5.6.15 y el gestor de aplicación PHPMYADMIN. Posteriormente, se configuraron los puertos 3306 y 8080, librándolos y activando el servidor y la aplicación de MySQL luego se implementó la estructura de la base de datos, se desarrollaron las diversas pruebas de integridad de la información y se codificaron los formularios.

La implementación de la base de datos en el servidor local también se diseñó con la ruta de creación y se visualiza en los círculos numerados que acompañan a las entidades, las reglas de integridad referencial y la lógica de construcción de las tablas se basa, primero, en la creación de las entidades fuertes marcadas con línea gruesa, es decir, las que aportan las claves foráneas para la integridad de la información, seguido las de líneas 
medianas, excepto las de generalización, que fueron las últimas entidades creadas dentro del modelo (Figura 4).

Un aspecto importante dentro de la integridad referencial por resaltar de la fase de codificación es la lógica requerida para la construcción de llaves foráneas y principales, dado que en las entrevistas no se tienen en cuenta estos aspectos, pero sí necesita un tiempo importante de cálculo para el diseño. En este caso, se requería relacionar por materia o asignatura ocho momentos (Campo actiity_w1-8), por lo que implicó tener todos estos aspectos en cuenta al momento del diseño de las llaves principales y foráneas de la base de datos (Figura 5).

Figura 5. Base de datos Paponline

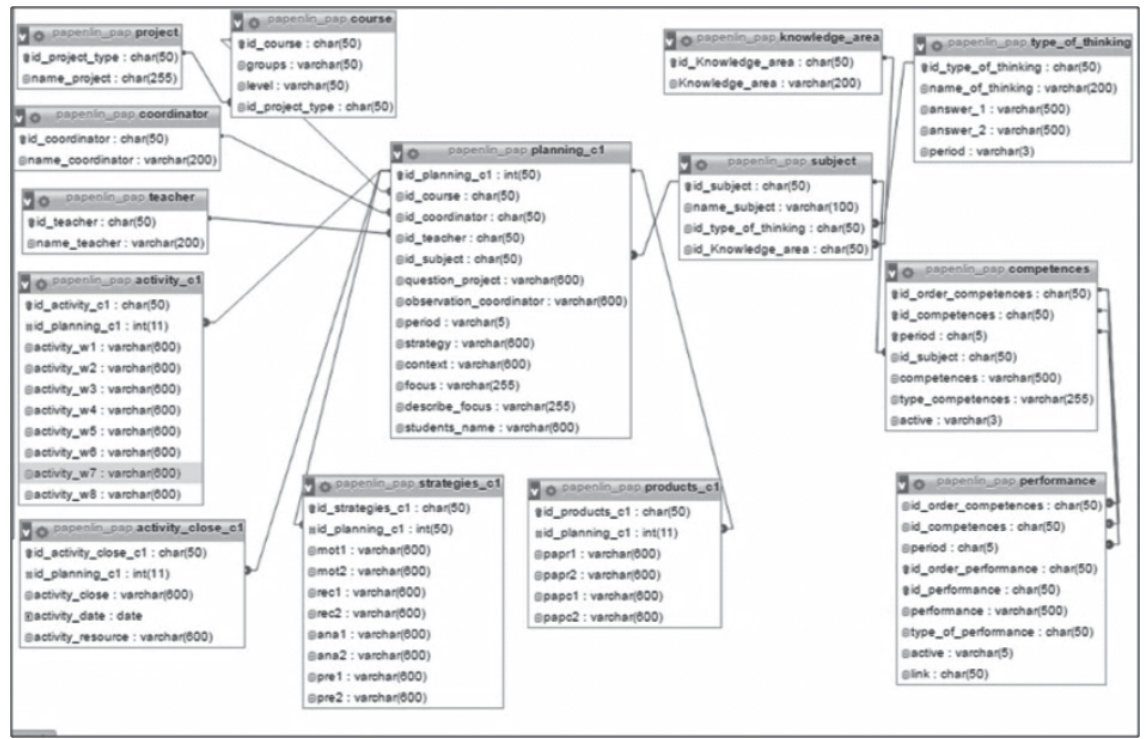

Fuente. Elaboración propia. 


\subsection{Fase de comprobación}

En esta fase se realizaron tres pasos que conforman las estrategias para configurar el indicador de efectividad. El primer paso fue la entrevista con el rector del Colegio IED Cafam Bellavista, mediada por la figura del prototipo, el objetivo de este consistió en unificar las observaciones ${ }^{5}$ por módulo, incluyendo todas las respuestas de los aspectos orientadores que tocan tangencialmente al módulo. La matriz de este primer momento estaba compuesta por el nombre del módulo y observaciones (Tabla 3):

Tabla 3. Análisis de requerimientos relacionados en tiempo de prototipo.

\begin{tabular}{|l|c|c|}
\hline \multicolumn{1}{|c|}{ Módulo } & Observaciones & Porcentaje \\
\hline Datos generales & 6 & 18 \\
\hline Pregunta problémica por tipo de pensamiento & 4 & 12 \\
\hline Competencias-desempeños & 8 & 24 \\
\hline Estrategias de enseñanza -aprendizaje & 3 & 9 \\
\hline Ruta metodológica & 4 & 12 \\
\hline Productos de aprendizaje & 3 & 9 \\
\hline Cierre de proyecto & 2 & 6 \\
\hline Enfoque diferencial & 2 & 6 \\
\hline Observaciones de coordinación & 2 & 6 \\
\hline Total & 34 & 100 \\
\hline
\end{tabular}

Fuente. Elaboración propia.

El segundo paso fue la revisión de la aplicación en ejecución por parte del grupo primario, cuyo objetivo consistió en unificar las observaciones con la aplicación web desarrollada. En ambos pasos, se tuvieron en cuenta todas las observaciones sobre la funcionalidad o calidad de los módulos. Con esta información se realizó la comparación de los dos resultados de los pasos descritos y se construyó el indicador de efectividad utilizado para medir la calidad del producto del trabajo realizado tabla 4). 
Tabla 4. Análisis de requerimientos relacionados con la aplicación web desarrollada

\begin{tabular}{|l|c|c|}
\hline \multicolumn{1}{|c|}{ Módulo } & Observaciones & Porcentaje \\
\hline Datos generales & 1 & 17 \\
\hline Pregunta problémica por tipo de pensamiento & 0 & 0 \\
\hline Competencias-desempeños & 1 & 17 \\
\hline Estrategias de enseñanza -aprendizaje & 1 & 17 \\
\hline Ruta metodológica & 1 & 17 \\
\hline Productos de aprendizaje & 0 & 0 \\
\hline Cierre de proyecto & 0 & 0 \\
\hline Enfoque diferencial & 2 & 33 \\
\hline Observaciones de coordinación & 0 & 0 \\
\hline Total & 6 & 100 \\
\hline
\end{tabular}

Fuente. Elaboración propia.

Teóricamente, la expresión se conjugaría a partir de una regla de tres, en donde el denominador se obtiene de sumar las observaciones captadas por medio del prototipo creado más las observaciones ${ }^{5}$ captadas con la aplicación desarrollada, y el numerador corresponde con lo captado en el paso 2. Matemáticamente, se podría establecer que, a más observaciones en desarrollo, se disminuye la efectividad. Por lo tanto, surge reprocesos para la etapa de codificación, porque se tendrían que volver a realizar los desarrollos para cumplir con las observaciones de los líderes, captadas en el segundo paso (Figura 6).

El término observaciones hace referencia a la lista de respuestas elicitadas en la fase 1, pero visto desde las relaciones directas o tangenciales con los módulos de la aplicación, es decir, si inciden directamente en el requerimiento o lo mencionan en su contenido. 
Figura 6. Indicador de efectividad de la metodología

$$
\text { Efectividad promedio }=\frac{\text { Observaciones en desarrollo }}{\text { Total de observaciones }} * 100
$$

Fuente. Elaboración propia.

A partir de lo anterior, se muestra el consolidado final validación, en donde se procedió a calcular el indicador de efectividad del modelo; vale la pena destacar que las observaciones realizadas en tiempo de desarrollo no fueron de carácter estructural, es decir, no se tuvo que modificar las relaciones entre las entidades, tan solo se trata de agregar algunos campos requeridos (Tabla 5).

Tabla 5. Consolidado de efectividad metodológica.

\begin{tabular}{|l|c|c|c|c|}
\hline \multicolumn{1}{|c|}{ Módulo } & \multicolumn{4}{c|}{ Observaciones } \\
\hline & $\begin{array}{c}\text { Análisis } \\
\text { prototipo }\end{array}$ & $\begin{array}{c}\text { Verificación } \\
\text { Desarrollo }\end{array}$ & Total & Efectividad \\
\hline Datos generales & 6 & 1 & 7 & $86 \%$ \\
\hline $\begin{array}{l}\text { Pregunta problémica por } \\
\text { tipo de pensamiento }\end{array}$ & 4 & 0 & 4 & $100 \%$ \\
\hline Competencias-desempeños & 8 & 1 & 9 & $89 \%$ \\
\hline $\begin{array}{l}\text { Estrategias de enseñanza } \\
\text {-aprendizaje }\end{array}$ & 3 & 1 & 4 & $75 \%$ \\
\hline Ruta metodológica & 4 & 1 & 5 & $80 \%$ \\
\hline Productos de aprendizaje & 3 & 0 & 3 & $100 \%$ \\
\hline Cierre de proyecto & 2 & 0 & 2 & $100 \%$ \\
\hline Enfoque diferencial & 2 & 2 & 4 & $50 \%$ \\
\hline $\begin{array}{l}\text { Observaciones de } \\
\text { coordinación }\end{array}$ & 2 & 0 & 2 & $100 \%$ \\
\hline Total & 34 & 6 & & $86.6 \%$ \\
\hline
\end{tabular}

Fuente. Elaboración propia. 
Figura 7. Modelo o prototipo de pantalla.

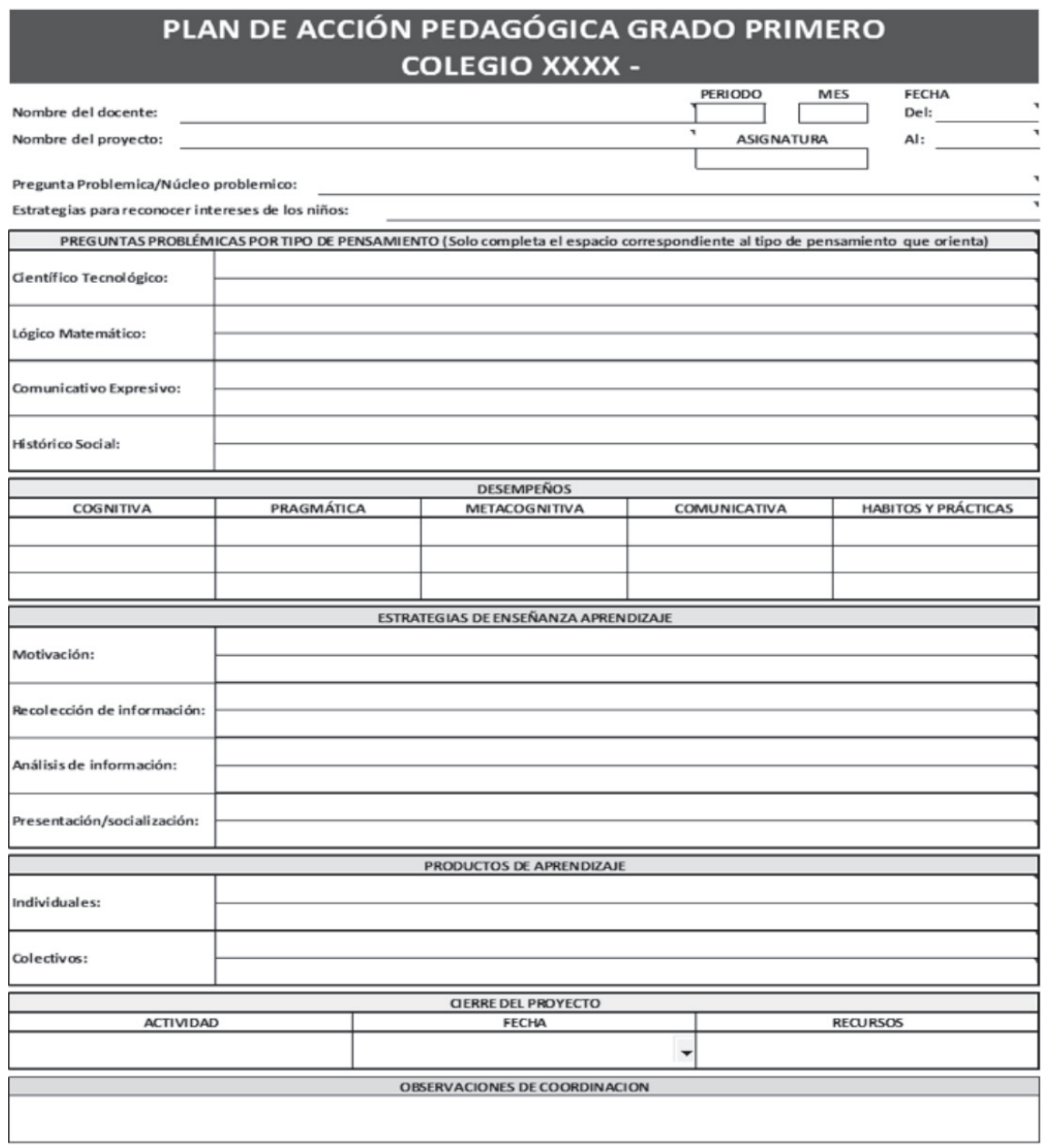

Fuente. Elaboración propia. 


\subsection{Fase evaluación de impresiones}

En esta última fase se aplicó una encuesta de verificación de impresiones después de un proceso de planeación pedagógica, evidenciando el acierto o desacierto en las expectativas proceso de levantamiento de requerimientos y funcionamiento de la herramienta creada, obteniendo un indicador de satisfacción. Es importante ratificar que los porcentajes de confianza (95\%) y error (10 \%) del estudio fueron aplicados a cabalidad y expresados en el tamaño de la muestra de forma completa.

Por otra parte, el proceso de validación del instrumento se realizó con el aval de expertos y cumpliendo con los estándares de los autores seleccionados, el índice de Alfa de Cronbach $(0,93)$ y la muestra inicial realizada con cinco personas. El resultado final de este ejercicio permite concluir, que no se puede descartar la hipótesis nula que dice que la expectativa es igual resultado en términos estadísticos.

Como la hipótesis nula no se descarta, esto significa que las expectativas de los usuarios son iguales a los resultados del producto entregado. Los resultados se visualizaron en términos de satisfacción de uso de la herramienta, los cuales se pueden visualizar en la tabla 4 , que entrega un total de expectativas seleccionadas entre 1 y 2 (insatisfecho), más 4 y 5 (satisfecho) y descartando la opción 3 que se interpreta como indeciso.

El primer par de opciones (Rango 1-2) de la escala del formato tiene un total de 20 puntos, mientras el segundo par de opciones (Rango 4-5) tiene 218 puntos. Ahora bien, para traducir estas cifras a un indicador que señale el nivel de satisfacción, se formula el indicador que se estructura en términos de respuestas de Rango 4-5 (numerador), dividido por la suma de los Rangos 1-2 más Rango 4-5, lo que genera un total de 238 puntos, y al realizar el cálculo se obtiene un $95 \%$ (218/238) de satisfacción (Tabla 6). 
Tabla 6. Resultados encuestas de satisfacción.

\begin{tabular}{|c|c|c|}
\hline Escala & $\begin{array}{c}\text { Sumatoria de } \\
\text { expectativa y } \\
\text { funcionamiento }\end{array}$ & $\begin{array}{c}\text { Total de sumatoria } \\
\text { Expectativas y } \\
\text { funcionamiento }\end{array}$ \\
\hline 1 & 20 & \multirow{2}{*}{238} \\
\cline { 1 - 1 } 2 & 218 & \\
\hline 4 & & \\
\hline 5 & & \\
\hline
\end{tabular}

Fuente. Elaboración propia.

\section{Conclusiones y recomendación para futuros trabajos de investigación}

$\checkmark$ n este apartado se mostrará la experiencia adquirida en el desarrollo del

Cproceso, mostrando el recorrido de la metodología con las estrategias empleadas para obtener resultados favorables en los indicadores de efectividad y satisfacción, de la misma manera las estrategias que podrían mitigar bajos resultados en procesos similares, finalmente se muestran los resultados en términos cuantitativos y operativos de cada fase de la presente metodología.

De manera general, los resultados obtenidos en el indicador de efectividad correspondieron a un $86,6 \%$, esto se asocia con el uso de las herramientas que canalizaban el lenguaje y el entendimiento entre especialista e interesado. El otro aspecto por señalar como resultado positivo, es el indicador de satisfacción de los docentes que registra un 92 $\%$. Consideramos que el factor que determinó este resultado consistió en solo enfocar el cumplimiento de los requerimientos funcionales y generar una estructura automática para los requerimientos no funcionales. 
En cuanto a los módulos que registraron promedios bajos en el indicador de efectividad, las causas están enmarcadas en la aleatoriedad de los requerimientos por los procesos de actualización o innovación de las tareas pedagógicas, y como estrategia para mitigar esos cambios en las estructuras elicitadas se recomienda generar un conjunto de preguntas que pertenezcan al aspecto sistémico, las cuales son las encargadas de proyectar este tipo de requerimientos; por otra parte, se recomienda que se inicie con la búsqueda de información documental o gráfica, que oriente el entendimiento y posterior proyección de los requerimientos.

En la fase de elicitación, los resultados se expresan en términos cuantitativos y operacionales, dentro del primero se encuentra la formulación de cinco aspectos orientadores con un total de 25 preguntas. Los aspectos orientadores fueron: la fundamentación, el tiempo, la organización institucional, el aspecto pedagógico (asociado al negocio) y el aspecto sistémico. En la parte operacional se logró el entendimiento de todo el universo de las entidades y su comportamiento, características y demás aspectos necesarios en el diseño, implantación y codificación de una herramienta.

Se identifican como posibles estrategias para mitigar observaciones en la fase de desarrollo: generar un conjunto de preguntas que indaguen por este aspecto de requerimientos aleatorios, ejecutar entrevistas con diferentes interesados, desarrollar un encuentro grupal con los líderes principales con el ánimo de retroalimentar las respuestas encontradas y unificar los criterios y las expectativas que aseguren la calidad de los requerimientos identificados.

Los resultados de la fase de modelado en términos cuantitativos se visualizan desde dos momentos: en el primer momento se logró el desarrollo del prototipo, posteriormente se expresan en la cantidad de requerimientos y entidades generadas en los procesos de análisis relacionados con los aspectos orientadores. En conclusión, de esta fase se puede apreciar que en el aspecto de fundamentación se configuraron 
en total 12 requerimientos y 15 entidades, en términos operativos se materializó todo el entendimiento del modelo relacional, insumo para la siguiente fase.

La fase de comprobación entrega resultados en términos cuantitativos a través de una estructura que compara dos hitos: el primer hito es la fase de elicitación mediada a través de la entrevista y el formato de preguntas, del cual se extrae un total de 34 observaciones, el segundo hito corresponde a la presentación del desarrollo de la aplicación a los líderes de los interesados, de la cual se extrae un total de seis observaciones; es importante señalar que esta fase es propuesta por los autores de este artículo.

Posteriormente, en la subfase de validación se comparan las observaciones de cada módulo, obteniendo cuatro módulos con efectividad $100 \%$ (sin ninguna observación), tres módulos con efectividad entre 80 y $90 \%$ de efectividad, y dos módulos con efectividad entre 60 y $70 \%$. En promedio, la efectividad de este proceso se estima en el $86,6 \%$, que en términos operacionales esta fase entrega la visual que tienen los líderes sobre el desarrollo de la metodología antes y después de la materialización de la aplicación, obteniendo de esta los resultados en cada módulo y la manera de proyectar las mejoras en los procesos de levantamiento de requerimientos y el modelamiento de estos.

La fase de evaluación e impresión de uso de la herramienta entrega los resultados en términos de satisfacción de empleo por parte de los docentes, comparando las expectativas versus la calidad en el desarrollo de la herramienta en cada uno de los módulos, obteniendo un $92 \%$ de efectividad; en términos operacionales esta fase entrega la visual que tienen los docentes sobre la metodología empleada a través del uso de la aplicación y de esta manera enfocar en cómo a través de la estructura se minimizan las acciones técnicas que tengan que desarrollar los funcionarios para realizar una determinada actividad. 


\subsection{Recomendaciones o sugerencias para otros estudios}

El origen de este trabajo estuvo relacionado con la preparación de forma autodidacta, explorando varios campos del mundo de la informática, orientado por recursos bibliográficos y el pensamiento fijado en el querer aportar una herramienta para el contexto descrito inicialmente, lo que en su conjunto fue el motor que inició esta experiencia.

Por lo anterior, y de acuerdo con los avances en el desarrollo de trabajo, los campos que se podrían complementar en este ámbito estarían relacionados con el levantamiento de requerimientos por medio de técnicas de elicitación que involucren rastreo de información escrita o gráfica, mediante la comparación de diversos recursos ya sean gráficos o digitales y la posterior materialización en un software. 


\section{Referencias}

Alvarado, J., \& Cuervo, C. (2013). Extracción de funciones de un cargo usando minería de texto en correos electrónicos. Información Tecnológica, 24(5), 61-67. http://dx.doi.org/10.4067/S071807642013000500008

Anaima, R. (2015). Ingeniería de requistos para multitudes. Revista Antioqueña de las Ciencias computacionales y la Ingeniería de software, 5(2), 17-20.

Manies, M., \& Nikual, U. (2011). La elicitación de requisitos en el contexto de un proyecto de software. Ing. USBMed, 2(2), 25-29.

Mansilla, D., Pollo-Cattaneo, F., Britos, P., Pesado, P., \& García-Martínez, R. (2012). Modelo de proceso para elicitación de requerimientos en proyectos de explotación de información. Requirements Enginneering \& Software Testing, 38-45.

Pérez, H., Salamando, C., \& Valencia, L. (2012). Levantamiento de requerimientos basados en el conocimiento del proceso. Revista Cientifica, 2(16), 42-51.

Schach, S. (2006). Ingeniería del software orientada a objetos (VI Edición). México: McGraw-Hill / Interamericana.

Terstine, M. (2015). El progreso de la investigación en ingeniería de requisitos. Revista Antioqueña de las Ciencias Computacionales y la Ingenieria del software, 5(1), 18-24. 